Research Article

\title{
Iterative Construction of Fixed Points for Operators Endowed with Condition $(E)$ in Metric Spaces
}

\author{
Junaid Ahmad $\mathbb{D}^{1},{ }^{1}$ Kifayat Ullah $\mathbb{D}{ }^{2}$ Hüseyin Işik $\mathbb{D}^{\mathbb{D}},{ }^{3}$ Muhammad Arshad $\mathbb{D}^{1}$, \\ and Manuel de la Sen ${ }^{4}{ }^{4}$ \\ ${ }^{1}$ Department of Mathematics and Statistics, International Islamic University, H-10 Islamabad-44000, Pakistan \\ ${ }^{2}$ Department of Mathematics, University of Lakki Marwat, Lakki Marwat, 28420 Khyber Pakhtunkhwa, Pakistan \\ ${ }^{3}$ Department of Engineering Science, Bandırma Onyedi Eylül University, 10200 Bandırma, Balıkesir, Turkey \\ ${ }^{4}$ Institute of Research and Development of Processes, University of the Basque Country, Campus of Leioa (Bizkaia), P.O. Box 644- \\ Bilbao, Barrio Sarriena, 48940 Leioa, Spain
}

Correspondence should be addressed to Junaid Ahmad; ahmadjunaid436@gmail.com and Hüseyin Işik; isikhuseyin76@gmail.com Received 22 April 2021; Accepted 14 June 2021; Published 9 July 2021

Academic Editor: Ming Mei

Copyright (C) 2021 Junaid Ahmad et al. This is an open access article distributed under the Creative Commons Attribution License, which permits unrestricted use, distribution, and reproduction in any medium, provided the original work is properly cited.

We consider the class of mappings endowed with the condition $(E)$ in a nonlinear domain called 2-uniformly convex hyperbolic space. We provide some strong and $\Delta$-convergence theorems for this class of mappings under the Agarwal iterative process. In order to support the main outcome, we procure an example of mappings endowed with the condition $(E)$ and prove that its Agarwal iterative process is more effective than Mann and Ishikawa iterative processes. Simultaneously, our results hold in uniformly convex Banach, CAT(0), and some CAT $(\kappa)$ spaces. This approach essentially provides a new setting for researchers who are working on the iterative procedures in fixed point theory and applications.

\section{Introduction}

Choose a metric space $\mathscr{Z}$ and $\varnothing \neq S \subseteq \mathscr{Z}$. Then, a self-map $\mathscr{L}: S \longrightarrow S$ is called nonexpansive if

$$
q\left(\mathscr{L} s, \mathscr{L}^{\prime}\right) \leq q\left(s, s^{\prime}\right), \quad \text { for all } \quad s, s^{\prime} \in S
$$

As many know, Browder [1] (see also Kirk [2] and Gohde [3]) achieved an elementary fixed point theorem for nonexpansive map $\mathscr{L}: S \longrightarrow S$ in the case when $S$ is closed bounded convex in any given uniformly convex Banach space (UCBS). This remarkable theorem began the research of different results for nonexpansive maps. Among other things, Kirk [4] obtained this theorem in the nonlinear setting of CAT(0) metric spaces. The class of nonexpansive mappings is very important because of its useful applications in many fields of applied sciences. Thus, it is very natural and essential to study the extension of these maps. In 2008, Suzuki [5] considered an extension of nonexpansive maps by weakening the inequality of the nonexpansive maps in the following way: a self-map $\mathscr{L}: S \longrightarrow S$ is said to hold condition $(C)$ if any given $s, s^{\prime} \in S$,

$$
\frac{1}{2} q(s, \mathscr{L} s) \leq q\left(s, s^{\prime}\right) \Rightarrow q\left(\mathscr{L} s, \mathscr{L}^{\prime}\right) \leq q\left(s, s^{\prime}\right)
$$

Obviously, a nonexpansive map $\mathscr{L}$ has condition $(C)$, but an example in [5] shows the inverse is not valid in general. In [6], Nanjaras et al. improved the main outcome of Suzuki [5] to the nonlinear setting of CAT(0) spaces. In [7], Phuengrattana used Ishikawa iteration [8] for reckoning of fixed points for the class of Suzuki mappings in a Banach space. Also, Basarir and Sahin [9] used Agarwal et al.'s [8] iteration for reckoning fixed points of Suzuki mappings in the nonlinear setting of $\mathrm{CAT}(0)$ spaces.

In [10], Garcia-Falset et al. suggested a new type of maps which is properly more general than Suzuki maps. A selfmap $\mathscr{L}$ of $S$ is called Garcia-Falset nonexpansive or said to be endowed with the condition $(E)$ if any given $s, s^{\prime} \in S$, there 
is a $\mu \geq 1$ such that

$$
q\left(s, \mathscr{L} s^{\prime}\right) \leq \mu q(s, \mathscr{L} s)+q\left(s, s^{\prime}\right)
$$

Remark 1. If $\mathscr{L}$ is nonexpansive, then it is easy to show $q(s$, $\left.\mathscr{L} s^{\prime}\right) \leq q(s, \mathscr{L} s)+q\left(s, s^{\prime}\right)$ for each $s, s^{\prime} \in S$. Hence, every nonexpansive map $\mathscr{L}$ has condition $(E)$ with $\mu=1$. Similarly, if $\mathscr{L}$ is Suzuki map, then, it satisfies $q\left(s, \mathscr{L} s^{\prime}\right) \leq 3 q(s, \mathscr{L} s)+q($ $\left.s, s^{\prime}\right)$ for every $s, s^{\prime} \in S$. Hence, every Suzuki map $\mathscr{L}$ has condition $(E)$ with $\mu=3$. Interestingly, the example constructed in this paper shows that there exists maps satisfying condition $(E)$ but not the converse.

After establishing the existence of a fixed point result for an operator, a natural question arises on how to compute it by any appropriate numerical method. To work on such type of problems is not easy. The well-known Banach Contraction Theorem (BCT) essentially suggests the Picard iteration $y_{k+1}=\mathscr{L} y_{k}$ to compute the unique fixed point of a given contraction in a metric space. Bagherboum [11] used Ishikawa iteration [12] for computing fixed points of Garcia-Falset mappings in the nonlinear setting of Busemann spaces. We know that the Picard iteration fails for solving nonexpansive problems. Now, we discuss some other methods which are different from the Picard iteration process.

One of the elementary iterative processes is due to Mann [13] stated as follows:

$$
\left\{\begin{array}{l}
y_{1} \in S \\
y_{k+1}=\left(1-\alpha_{k}\right) y_{k} \oplus \alpha_{k} \mathscr{L} y_{k}
\end{array}\right.
$$

where $\alpha_{k} \in[0,1]$.

The Mann iteration (4) was extended to the setting of two steps by Ishikawa [12] as follows:

$$
\left\{\begin{array}{l}
y_{1} \in S, \\
y_{k+1}=\left(1-\alpha_{k}\right) y_{k} \oplus \alpha_{k} \mathscr{L} w_{k}, \\
w_{k}=\left(1-\beta_{k}\right) y_{k} \oplus \beta_{k} \mathscr{L} y_{k},
\end{array}\right.
$$

where $\alpha_{k}, \beta_{k} \in[0,1]$.

In 2007, Agarwal et al. [8] studied the following iteration for contractions and observed that it is better than the above iterative processes:

$$
\left\{\begin{array}{l}
y_{1} \in S \\
y_{k+1}=\left(1-\alpha_{k}\right) \mathscr{L} y_{k} \oplus \alpha_{k} \mathscr{L} w_{k} \\
w_{k}=\left(1-\beta_{k}\right) y_{k} \oplus \beta_{k} \mathscr{L} y_{k}
\end{array}\right.
$$

where $\alpha_{k}, \beta_{k} \in[0,1]$.

In this research, we consider the Agarwal iterative process (6) for the larger class of maps due to Garcia-Falset et al. [10]. We use a very general ground space called 2uniformly convex hyperbolic metric space for establishing the main outcome. To support our results, we provide an example of maps with condition $(E)$ and prove that its Agarwal iterative process (6) is more effective than Mann (4) and Ishikawa iterative (5) processes. Simultaneously, our results hold in uniformly convex Banach, CAT(0), and some $\mathrm{CAT}(\kappa)$ spaces. In this way, we extend the corresponding results of Bagherboum [11], Phuengrattana [7], Basarir and Sahin [9], and Nanjaras et al. [6] as we consider the general setting of domain, larger class of maps, and faster iteration process.

Now, we present some well-known definitions and results which will be either used in the main results or to understand the given concept herein.

Definition 2 (see [14]). A given metric space $(\mathscr{Z}, q)$ is called Kolenbach-hyperbolic space or simply hyperbolic space when there is a map $C: \mathscr{Z} \times \mathscr{Z} \times[0,1] \longrightarrow \mathscr{Z}$ such that for all $s, s^{\prime}, u, p \in \mathscr{Z}$ and $i, j \in[0,1]$, we have

(C1) $q\left(u, C\left(s, s^{\prime}, i\right)\right) \leq(1-i) q(u, s)+i q\left(u, s^{\prime}\right)$;

(C2) $q\left(C\left(s, s^{\prime}, i\right), C\left(s, s^{\prime}, j\right)=|i-j| q\left(s, s^{\prime}\right)\right.$;

(C3) $C\left(s, s^{\prime}, i\right)=C\left(s^{\prime}, s, 1-i\right)$;

(C4) $q\left(C(s, u, i), C\left(s^{\prime}, p, i\right)\right) \leq(1-i) q\left(s, s^{\prime}\right)+i q(u, p)$.

Notice that, for given $s, s^{\prime} \in \mathscr{Z}$ and $i \in[0,1]$, we shall use $(1-i) s \oplus i s^{\prime}$ for $C\left(s, s^{\prime}, i\right)$. Also, the property $(C 1)$ gives

$$
\begin{aligned}
q\left(s,(1-i) s \oplus i s^{\prime}\right) & =i q\left(s, s^{\prime}\right) \text { and } q\left(s^{\prime},(1-i) s \oplus i s^{\prime}\right) \\
& =(1-i) q\left(s, s^{\prime}\right) .
\end{aligned}
$$

Keep in mind that $\varnothing \neq S \subseteq \mathscr{Z}$ is said to be convex in $\mathscr{Z}$ if for every $s, s^{\prime} \in S,\left[s, s^{\prime}\right]=\left\{(1-\sigma) s \oplus \sigma s^{\prime}: \sigma \in[0,1]\right\} \subseteq S$.

Definition 3. A given hyperbolic space $(\mathscr{Z}, q, C)$ is said to be a uniformly convex hyperbolic space (UCHS), if there exist $r$ $>0, \sigma \in(0,1]$ and $\varepsilon \in(0,2]$ such that for each $s, s^{\prime}, p \in \mathscr{Z}$ satisfying $q(s, p) \leq r, q\left(s^{\prime}, p\right) \leq r$ and $q\left(s, s^{\prime}\right) \geq 2 \varepsilon$ follows that:

$$
q\left(\frac{1}{2} s \oplus \frac{1}{2} s^{\prime}, p\right) \leq(1-\sigma) r
$$

Moreover, if $f:(0, \infty) \times(0,2] \longrightarrow(0,1]$ is given such that $\sigma=f(r, \varepsilon)$ for all $r \in(0, \infty)$ and $\varepsilon \in(0,2]$, then, $f$ is called a modulus of uniform convexity (MUC). Also, $f$ is called monotone modulus of uniform convexity (MMUC) iff nonincreasing in the variable $r$ for every chosen fixed $\varepsilon$.

Definition 4. Suppose a pair $(\mathscr{Z}, q)$ is a given hyperbolic space. For any $r \in(0, \infty)$ and $\varepsilon \in(0,2]$, define:

$$
\varphi(r, \varepsilon)=\inf \left\{\frac{1}{2} q^{2}(s, p)+\frac{1}{2} q^{2}\left(s^{\prime}, p\right)-q^{2}\left(\frac{1}{2} s \oplus \frac{1}{2} s^{\prime}, p\right)\right\}
$$

and keeping in mind that infimum is taken over every $s, s^{\prime}$, $p \in \mathscr{Z}$ satisfying $q(s, p) \leq r, q\left(s^{\prime}, p\right) \leq r$, and $q\left(s, s^{\prime}\right) \geq r \varepsilon$. Then, $(\mathscr{Z}, q)$ is called 2 -uniformly convex hyperbolic space 
(2-UCHS) provided that

$$
c_{M}=\inf \left\{\frac{\varphi(r, \varepsilon)}{r^{2} \varepsilon^{2}}: r \in(0, \infty), \varepsilon \in(0,2]\right\}>0 .
$$

The following remark suggests that 2-UCHS is a more general domain than many other linear and nonlinear spaces.

Remark 5. Any given UCBS, CAT(0) space, and CAT $(\kappa)$ spaces having $\kappa>0$ and $\operatorname{diam}(\mathscr{Z}) \leq\left(\pi / 2-\varepsilon / \kappa^{1 / 2}\right), \varepsilon \in(0, \pi /$ 2) can be considered as a 2-UCHS (see [15-17]).

Definition 6. Choose a complete 2-UCHS $\mathscr{Z}$ such that $\left\{y_{k}\right\}$ $\subseteq \mathscr{Z}$ is bounded and $\varnothing \neq S \subseteq \mathscr{Z}$ is closed as well as convex. The asymptotic radius of $\left\{y_{k}\right\}$ corresponding to $S$ is $r(S,\{$ $\left.\left.y_{k}\right\}\right)=\inf \left\{\limsup _{k \rightarrow \infty} q\left(y_{k}, u\right): u \in S\right\}$. Moreover, the asymptotic center of $\left\{y_{k}\right\}$ corresponding to $S$ is the set $A(S$, $\left.\left\{y_{k}\right\}\right)=\left\{u \in S: \limsup _{k \rightarrow \infty} q\left(y_{k}, u\right)=r\left(S, y_{k}\right)\right\}$. In addition, if the underlying space has MMUC, then the set $A\left(S, y_{k}\right)$ is singleton (see, e.g., [18]).

Definition 7. Choose a complete 2-UCHS $\mathscr{Z}$ such that $\varnothing$ $\neq S \subseteq \mathscr{Z}$ is closed as well as convex. Fix an element $w \in S$ . Assume that $\left\{y_{k}\right\}$ is a bounded sequence in $\mathscr{Z}$. Then, $\left\{y_{k}\right\}$ is called $\Delta$-convergent to $w$ iff the asymptotic center $A\left(S,\left\{z_{k}\right\}\right)=\{w\}$ for all subsequence $\left\{z_{k}\right\}$ of $\left\{y_{k}\right\}$. When $w$ is $\Delta$-limit of $\left\{y_{k}\right\}$, then we shall write $\Delta-\lim _{k \rightarrow \infty} y_{k}$ $=w$.

Lemma 8 (see [19]). Any bounded sequence in a given 2UCHS having MMUC has a $\Delta$-convergent subsequence.

Lemma 9 (see [20]). Choose a complete 2-UCHS $\mathscr{Z}$ endowed with MMUC such that $\varnothing \neq S \subseteq \mathscr{Z}$ and $\left\{y_{k}\right\} \subseteq S$ is bounded. Then, the asymptotic center of $\left\{y_{k}\right\}$ is always in $S$.

Mappings endowed with condition (E) enjoy the following useful properties which we have combined in a proposition form. For details, we refer the reader to [10].

Proposition 10. Choose a complete 2-UCHS $\mathscr{Z}$ such that $\varnothing$ $\neq S \subseteq \mathscr{Z}$. Suppose $\mathscr{L}: S \longrightarrow S$.

(i) When the map $\mathscr{L}$ has condition (E) with nonempty fixed point set, then for each $s \in S$ and $\omega \in F_{\mathscr{L}}$, we have $q(\mathscr{L} s, \mathscr{L} \omega) \leq q(s, \omega)$

(ii) When the map $\mathscr{L}$ has condition (E) then fixed point set of $\mathcal{S}$ is always closed in $S$

We now state an important characterization of a 2UCHS, which was proved in [15].
Lemma 11. Choose a 2-UCHS $(\mathscr{Z}, q)$. Then, the following inequality holds

$$
\begin{aligned}
q^{2}\left((1-\xi) s \oplus \xi s^{\prime}, g\right) \leq & (1-\xi) q^{2}(s, g)+\xi q^{2}\left(s^{\prime}, g\right) \\
& -4 c_{M} \xi(1-\xi) q^{2}\left(s, s^{\prime}\right),
\end{aligned}
$$

for each $\xi \in[0,1]$ and $s, s^{\prime}, g \in \mathscr{Z}$.

\section{Approximation Results}

We now want to show our desirable strong and $\Delta$-convergence results by considering the Agarwal iteration (6). It should be noted that $\mathscr{Z}$ will be considered complete and endowed with the MMUC. The notation $F_{\mathscr{L}}$ will simply denote the set of all fixed points of $\mathscr{L}$.

We suggest the following useful lemma which will provide a key role in main outcome.

Lemma 12. Choose a 2-UCHS $\mathscr{Z}$ such that $\varnothing \neq S \subseteq \mathscr{Z}$ is closed as well as convex. Furthermore, let $\mathscr{L}: S \longrightarrow S$ be endowed with condition (E) having $F_{\mathscr{L}} \neq \varnothing$. If $\left\{y_{k}\right\}$ is the Agarwal iterates (6), then, $\lim _{k \rightarrow \infty} q\left(y_{k}, \omega\right)$ exists for all $\omega \in F_{\mathscr{L}}$.

Proof. Assume that $\omega \in F_{\mathscr{L}}$. By using Proposition 10 (i), one has

$$
\begin{aligned}
q\left(w_{k}, \omega\right)= & q\left(\left(1-\beta_{k}\right) y_{k} \oplus \beta_{k} \mathscr{L} y_{k}, \omega\right) \leq\left(1-\beta_{k}\right) q\left(y_{k}, \omega\right) \\
& +\beta_{k} q\left(\mathscr{L} y_{k}, \omega\right) \leq\left(1-\beta_{k}\right) q\left(y_{k}, \omega\right)+\beta_{k} q\left(y_{k}, \omega\right) \\
\leq & q\left(y_{k}, \omega\right) .
\end{aligned}
$$

Hence,

$$
\begin{aligned}
q\left(y_{k+1}, \omega\right) & =q\left(\left(1-\alpha_{k}\right) \mathscr{L} y_{k} \oplus \alpha_{k} \mathscr{L} w_{k}, \omega\right) \\
& \leq\left(1-\alpha_{k}\right) q\left(\mathscr{L} y_{k}, \omega\right)+\alpha_{k} q\left(\mathscr{L} w_{k}, \omega\right) \\
& \leq\left(1-\alpha_{k}\right) q\left(y_{k}, \omega\right)+\alpha_{k} q\left(w_{k}, \omega\right) \leq q\left(y_{k}, \omega\right) .
\end{aligned}
$$

From the above observations, we have $q\left(y_{k+1}, \omega\right) \leq q\left(y_{k}\right.$, $\omega)$. Hence, the real sequence $\left\{q\left(y_{k}, \omega\right)\right\}$ is bounded below by 0 and nonincreasing as well, so $\lim _{k \longrightarrow \infty} q\left(y_{k}, \omega\right)$ exists for every $\omega \in F_{\mathscr{L}}$.

The existence of a fixed point for a self-map $\mathscr{L}: S \longrightarrow S$ having condition $(E)$ in the nonlinear domain 2-UCBS can be established by the following way. This result provides a fundamental key for establishing the main results in the sequel.

Theorem 13. Choose a 2 -UCHS $\mathscr{Z}$ such that $\varnothing \neq S \subseteq \mathscr{Z}$ is closed as well as convex. Furthermore, let $\mathscr{L}: S \longrightarrow S$ be endowed with condition (E). If $\left\{y_{k}\right\}$ is the Agarwal iterates (6) with restriction $\alpha_{k}, \beta_{k} \in[a, b] \subset(0,1)$. Then, $F_{\mathscr{L}} \neq \varnothing$ iff \{ $\left.y_{k}\right\}$ is bounded and $\lim _{k \longrightarrow \infty} q\left(y_{k}, \mathscr{L} y_{k}\right)=0$. 
Proof. Suppose that the iterative sequence $\left\{y_{k}\right\}$ is bounded having $\lim _{k \rightarrow \infty} q\left(\mathscr{L} y_{k}, y_{k}\right)=0$. We may fix an element, namely, $\omega \in A\left(S,\left\{y_{k}\right\}\right)$. By using condition $(E)$ of $\mathscr{L}$, we have

$$
\begin{aligned}
r\left(\mathscr{L} \omega,\left\{y_{k}\right\}\right)= & \underset{k \longrightarrow \infty}{\limsup q}\left(y_{k}, \mathscr{L} \omega\right) \leq \underset{k \longrightarrow \infty}{\limsup } q\left(\mathscr{L} y_{k}, y_{k}\right) \\
& +\underset{k \longrightarrow \infty}{\limsup } q\left(y_{k}, \omega\right)=\underset{k \longrightarrow \infty}{\limsup } q\left(y_{k}, \omega\right) \\
= & r\left(\omega,\left\{y_{k}\right\}\right) .
\end{aligned}
$$

It follows that $\mathscr{L} \omega \in A\left(S,\left\{y_{k}\right\}\right)$. Since $A\left(S,\left\{y_{k}\right\}\right)$ is singleton set, one has $\mathscr{L} \omega=\omega$. Thus, $F_{\mathscr{L}} \neq \varnothing$. Conversely, we suppose that $F_{\mathscr{L}} \neq \varnothing$ and $\omega \in F_{\mathscr{L}}$. Then, by the proof of Lemma 12, $\left\{y_{k}\right\}$ is bounded. By Lemma 11, we have

$$
\begin{aligned}
q^{2}\left(w_{k}, \omega\right) \leq & \left(1-\beta_{k}\right) q^{2}\left(y_{k}, \omega\right)+\beta_{k} q^{2}\left(\mathscr{L} y_{k}, \omega\right) \\
& -4 c_{M} \beta_{k}\left(1-\beta_{k}\right) q^{2}\left(y_{k}, \mathscr{L} y_{k}\right) \leq\left(1-\beta_{k}\right) q^{2}\left(y_{k}, \omega\right) \\
& +\beta_{k} q^{2}\left(y_{k}, \omega\right)-4 c_{M} \beta_{k}\left(1-\beta_{k}\right) q^{2}\left(y_{k}, \mathscr{L} y_{k}\right) \\
\leq & q^{2}\left(y_{k}, \omega\right)-4 c_{M} \beta_{k}\left(1-\beta_{k}\right) q^{2}\left(y_{k}, \mathscr{L} y_{k}\right) .
\end{aligned}
$$

Thus,

$$
\begin{aligned}
q^{2}\left(y_{k+1}, \omega\right) \leq & \left(1-\alpha_{y}\right) q^{2}\left(\mathscr{L} y_{k}, \omega\right)+\alpha_{y} q^{2}\left(\mathscr{L} w_{k}, \omega\right) \\
& -4 c_{M} \alpha_{k}\left(1-\alpha_{k}\right) q^{2}\left(\mathscr{L} y_{k}, \mathscr{L} w_{k}\right) \\
\leq & \left(1-\alpha_{k}\right) q^{2}\left(y_{k}, \omega\right)+\alpha_{k} q^{2}\left(w_{k}, \omega\right) \\
& -4 c_{M} \alpha_{k}\left(1-\alpha_{k}\right) q^{2}\left(\mathscr{L} y_{k}, \mathscr{L} w_{k}\right) \\
\leq & \left(1-\alpha_{k}\right) q^{2}\left(y_{k}, \omega\right)+\alpha_{k} q^{2}\left(w_{k}, \omega\right) \\
& -4 c_{M} \alpha_{k}\left(1-\alpha_{k}\right) q^{2}\left(\mathscr{L} y_{k}, \mathscr{L} w_{k}\right) \\
\leq & \left(1-\alpha_{k}\right) q^{2}\left(y_{k}, \omega\right)+\alpha_{k} q^{2}\left(w_{k}, \omega\right) \\
\leq & q^{2}\left(y_{k}, \omega\right)-4 c_{M} \alpha_{k} \beta_{k}\left(1-\beta_{k}\right) q^{2}\left(y_{k}, \mathscr{L} y_{k}\right) .
\end{aligned}
$$

Since $c_{M}>0$, it follows that

$$
\sum_{k=1}^{\infty} a^{2}(1-b) q^{2}\left(y_{k}, \mathscr{L} y_{k}\right) \leq \sum_{k=1}^{\infty} \alpha_{k} \beta_{k}\left(1-\beta_{k}\right) q^{2}\left(y_{k}, \mathscr{L} y_{k}\right)<\infty .
$$

Thus, $\lim _{k \longrightarrow \infty} q^{2}\left(y_{k}, \mathscr{L} y_{k}\right)=0$ and hence

$$
\lim _{k \longrightarrow \infty} q\left(y_{k}, \mathscr{L} y_{k}\right)=0
$$

In some sense, the notion of $\Delta$-convergence in nonlinear domains provides the analog of the notion of weak convergence in linear setting. To obtain the $\Delta$-convergence for operators having the condition $(E)$ in a 2 -UCHS by using the Agarwal iterative sequence (6), we shall propose the following techniques.

Theorem 14. Choose a 2-UCHS $\mathscr{Z}$ such that $\varnothing \neq S \subseteq \mathscr{Z}$ is closed as well as convex. Furthermore, let $\mathscr{L}: S \longrightarrow S$ be endowed with condition $(E)$ and $F_{\mathscr{L}} \neq \varnothing$. If $\left\{y_{k}\right\}$ is the Agarwal iterates (6) with restriction $\alpha_{k}, \beta_{k} \in[a, b] \subset(0,1)$, then, \{ $\left.y_{k}\right\} \Delta$-converges to a fixed point of $\mathscr{L}$.

Proof. It has been observed in Theorem 13 that the iterative sequence $\left\{y_{k}\right\}$ is bounded having $\lim _{k \longrightarrow \infty} q\left(\mathscr{L} y_{k}, y_{k}\right)=0$. We may consider the $\omega_{\Delta}\left(y_{k}\right)=\bigcup A\left(\left\{u_{k}\right\}\right)$ and notice that the union has been imposed on all possible subsequences \{ $\left.u_{k}\right\}$ of the iterative sequence $\left\{y_{k}\right\}$. We want to show that $\omega_{\Delta}\left(y_{k}\right) \subseteq F_{\mathscr{L}}$. Suppose $u \in \omega_{\Delta}\left(y_{k}\right)$. Then, one can find a subsequence $\left\{u_{k}\right\}$ of $\left\{y_{k}\right\}$ in such a way that $A\left(\left\{u_{k}\right\}\right)=\{u\}$. By Lemmas 8 and 9, one can find a subsequence $\left\{v_{k}\right\}$ of $\left\{u_{k}\right\}$ in such a way that $\Delta-\lim _{k \longrightarrow \infty} v_{k}=v \in S$. Keeping $\lim _{k \longrightarrow \infty} \rho($ $\left.v_{k}, \mathscr{L} v_{k}\right)=0$ in mind and applying condition $(E)$ of $\mathscr{L}$, one has

$$
q\left(v_{k}, \mathscr{L} v\right) \leq \mu q\left(v_{k}, \mathscr{L} v_{k}\right)+q\left(v_{k}, v\right)
$$

If we apply limsup on both of the sides of above, then, we have $v \in F_{\mathscr{L}}$. By Lemma $12, \lim _{k \longrightarrow \infty} q\left(y_{k}, v\right)$ exists. Next, we show that $u=v$. Assume on contrary that $u \neq v$. Then, using the property of uniqueness of asymptotic centers, we get

$$
\begin{aligned}
\limsup _{k \longrightarrow \infty} q\left(v_{k}, v\right) & =\limsup _{k \longrightarrow \infty} q\left(v_{k}, u\right) \leq \limsup _{k \longrightarrow \infty} q\left(u_{k}, u\right) \\
& <\limsup _{k \longrightarrow \infty} q\left(u_{k}, v\right)=\underset{k \longrightarrow \infty}{\limsup } q\left(y_{k}, v\right) \\
& =\limsup _{k \longrightarrow \infty} q\left(v_{k}, v\right) .
\end{aligned}
$$

The above strict inequality suggests a contradiction. So we must have $u=v \in F_{\mathscr{L}}$ and $\omega_{\Delta}\left(y_{k}\right) \subseteq F_{\mathscr{L}}$.

Finally, we show that $\left\{y_{k}\right\}$ is $\Delta$-convergent in $F_{\mathscr{L}}$, that is, we want to show $\omega_{\Delta}\left(y_{k}\right)$ is singleton. Assume that $\left\{u_{k}\right\}$ is a subsequence of the sequence $\left\{y_{k}\right\}$. In the view of Lemmas 8 and 9 , one can find a subsequence $\left\{v_{k}\right\}$ of $\left\{u_{k}\right\}$ in such a way that $\Delta-\lim _{k \rightarrow \infty} v_{k}=v \in S$. Suppose $A\left(\left\{u_{k}\right\}\right)=\{u\}$ and $A\left(\left\{y_{k}\right\}\right)=\{x\}$. We have already established that $u=v$ and $v \in F_{\mathscr{L}}$. Now, we have to show that $x=v$. Suppose not, then since $\lim _{k \longrightarrow q} q\left(y_{k}, v\right)$ exists and also the asymptotic centers are consist of exactly one point, so

$$
\begin{aligned}
\underset{k \longrightarrow \infty}{\limsup } q\left(v_{k}, v\right) & =\underset{k \longrightarrow \infty}{\limsup } q\left(v_{k}, x\right) \leq \underset{k \longrightarrow \infty}{\limsup } q\left(y_{k}, x\right) \\
& <\limsup _{k \longrightarrow \infty} q\left(y_{k}, v\right)=\underset{k \longrightarrow \infty}{\limsup } q\left(v_{k}, v\right) .
\end{aligned}
$$

which is obviously a contradiction and so $x=v \in F_{\mathscr{L}}$. Hence, $\omega_{\Delta}\left(y_{k}\right)=\{x\}$.

Normally, we are interested in a strong convergence. To obtain the strong convergence theorems, we essentially impose some other assumptions on the domain or the operator. We first take a compact domain and establish a strong convergence theorem as follows.

Theorem 15. Choose a 2-UCHS $\mathscr{Z}$ such that $\varnothing \neq S \subseteq \mathscr{Z}$ is compact as well as convex. Furthermore, let $\mathscr{L}: S \longrightarrow S$ be endowed with condition $(E)$ and $F_{\mathscr{L}} \neq \varnothing$. If $\left\{y_{k}\right\}$ is the 
Agarwal iterates (6) with restriction $\alpha_{k}, \beta_{k} \in[a, b] \subset(0,1)$. Then, $\left\{y_{k}\right\}$ converges strongly to a fixed point of $\mathscr{L}$.

Proof. Since $S$ is compact, we may select a subsequence $\left\{y_{k_{i}}\right\}$ of $\left\{y_{k}\right\}$ such that $y_{k_{i}} \longrightarrow g \in S$. By using condition $(E)$ of $\mathscr{L}$, we have

$$
q\left(y_{k_{i}}, \mathscr{L} g\right) \leq \mu q\left(y_{k_{i}}, \mathscr{L} y_{k_{i}}\right)+q\left(y_{k_{i}}, g\right) .
$$

In the view of Theorem 13, $\lim _{k \longrightarrow \infty} q\left(y_{k_{i}}, \mathscr{L} y_{k_{i}}\right)=0$. Hence, if we apply $k \longrightarrow \infty$ on both of the sides of the above inequality, we get $y_{k_{i}} \longrightarrow \mathscr{L} g$ by uniqueness of limits in metric spaces, $g=\mathscr{L} g$. Hence, $g$ is the fixed point of $\mathscr{L}$. Moreover, by Lemma 12, $\lim _{k \rightarrow \infty} q\left(y_{k}, g\right)$ exists. So $\left\{y_{k}\right\}$ is strongly convergent to $g$. This completes the proof.

The compactness of the domain in the above theorem provided us a significant help. Now, one thinks about how to replace the compactness of the domain by any other assumption. We replace it by the following condition, which was essentially defined by Senter and Dotson [21].

Definition 16. A self-map $\mathscr{L}$ on a subset $S$ of a 2 -UCHS $\mathscr{Z}$ is said to satisfy condition $(I)$, if there exists some nondecreasing function $\eta:[0, \infty) \longrightarrow[0, \infty)$ satisfying $\eta(0)=0, \eta(a)>0$ for every $a>0$ and $q(s, \mathscr{L} s) \geq \eta\left(\inf _{\omega \in F_{\mathcal{Y}}} q(s, \omega)\right)$ for each $s \in S$

Theorem 17. Choose a 2-UCHS $\mathscr{Z}$ such that $\varnothing \neq S \subseteq \mathscr{Z}$ is closed as well as convex. Furthermore, let $\mathscr{L}: S \longrightarrow S$ be endowed with conditions $(E)$ and $(I)$, and $F_{\mathscr{L}} \neq \varnothing$. If $\left\{y_{k}\right\}$ is the Agarwal iterates (6) with restriction $\alpha_{k}, \beta_{k} \in[a, b] \subset(0$ ,1). Then, $\left\{y_{k}\right\}$ converges strongly to a fixed point of $\mathscr{L}$.

Proof. Theorem 13 gives $\lim _{k \longrightarrow \infty} q\left(y_{k}, \mathscr{L} y_{k}\right)=0$. By condition $(I)$, one has

$$
\lim _{k \longrightarrow \infty} q\left(y_{k}, F_{\mathscr{L}}\right)=0 .
$$

Thus, one can choose a subsequence $\left\{y_{k_{j}}\right\}$ of $\left\{y_{k}\right\}$ and $\left\{\omega_{j}\right\}$ in $F_{\mathscr{L}}$ such that $q\left(y_{k_{j}}, \omega_{j}\right) \leq 1 / 2^{j}$ for every natural number $j$. Proof of Lemma 12 suggests that $\left\{y_{k}\right\}$ is nonicreasing, and so

$$
q\left(y_{k_{j+1}}, \omega_{j}\right) \leq q\left(y_{k_{j}}, \omega_{j}\right) \leq \frac{1}{2^{j}} .
$$

Now,

$$
\begin{aligned}
q\left(\omega_{j+1}, \omega_{j}\right) & \leq q\left(\omega_{j+1}, y_{k_{j+1}}\right)+\rho\left(y_{k_{j+1}}, \omega_{j}\right) \leq \frac{1}{2^{j+1}}+\frac{1}{2^{j}} \\
& \leq \frac{1}{2^{j-1}} \longrightarrow 0, \text { as } j \longrightarrow \infty .
\end{aligned}
$$

From the above, one can easily conclude that the
TABle 1: Strong convergence of Agarwal, Ishikawa, and Mann iterative schemes.

\begin{tabular}{lccc}
\hline & Agarwal (6) & Ishikawa (5) & Mann (4) \\
\hline$y_{1}$ & 0.9 & 0.9 & 0.9 \\
$y_{2}$ & 0.693125000000000 & 0.73812500000000 & 0.794999999999999 \\
$y_{3}$ & 0.533802517361111 & 0.60536501736111 & 0.702249999999999 \\
$y_{4}$ & 0.411102077606577 & 0.49648339271074 & 0.62032083333333 \\
$y_{5}$ & 0.316605697267843 & 0.40718533804957 & 0.54795006944444 \\
$y_{6}$ & 0.243830359909748 & 0.33394853071982 & 0.48402000000000 \\
$y_{7}$ & 0.187783242458271 & 0.27388417693063 & 0.42755326251928 \\
$y_{8}$ & 0 & 0.22462306455213 & 0.37767204855870 \\
$y_{9}$ & 0 & 0.18422211058060 & 0.33361030956019 \\
$y_{10}$ & 0 & 0.05526663317418 & 0.29468910677816 \\
\hline
\end{tabular}

sequence $\left\{\omega_{j}\right\}$ form a Cauchy sequence in the closed subset $F_{\mathscr{L}}$ of $S$. Hence, $\omega_{j} \longrightarrow g \in F_{\mathscr{L}}$ and so $g$ is a fixed point of $\mathscr{L}$. But Lemma 12 suggests that $\lim _{k \rightarrow \infty} q\left(y_{k}, g\right)$ exists. Thus, the element $g$ is the strong limit point of the sequence $\left\{y_{k}\right\}$.

\section{Numerical Observations}

This section is devoted to some numerical computations. We first suggest a nontrivial example of $\mathscr{L}$ endowed with the condition $(E)$, but not with $(C)$. This example shows that the class of Garcia-Falset is properly more general than the class of Suzuki mappings.

Example 1. Suppose $S=[0,1]$ and $\mathscr{L}$ is a self-map defined as follows:

$$
\mathscr{L} s= \begin{cases}0, & \text { if } s \in S_{1}=\left[0, \frac{1}{5}\right), \\ \frac{5 s}{6}, & \text { if } s \in S_{2}=\left[\frac{1}{5}, 1\right] .\end{cases}
$$

We fix the value of $\mu=6$. We shall prove that $q\left(s, \mathscr{L} s^{\prime}\right)$ $\leq 6 q(s, \mathscr{L} s)+q\left(s, s^{\prime}\right)$ for each $s, s^{\prime} \in S$.

Case I. Assume that $s, s^{\prime} \in S_{1}$. Then, $\mathscr{L} s=\mathscr{L} s^{\prime}=0$. Thus,

$$
\begin{aligned}
q\left(s, \mathscr{L} s^{\prime}\right)= & \left|s-\mathscr{L} s^{\prime}\right|=|s| \leq 6|s|=6|s-\mathscr{L} s| \leq 6|s-\mathscr{L} s| \\
& +\left|s-s^{\prime}\right|=6 q(s, \mathscr{L} s)+q\left(s, s^{\prime}\right) .
\end{aligned}
$$

Case II. Here, we choose $s, s^{\prime} \in S_{2}$. Then $\mathscr{L} s=5 s / 6$ and $\mathscr{L} s^{\prime}$ 


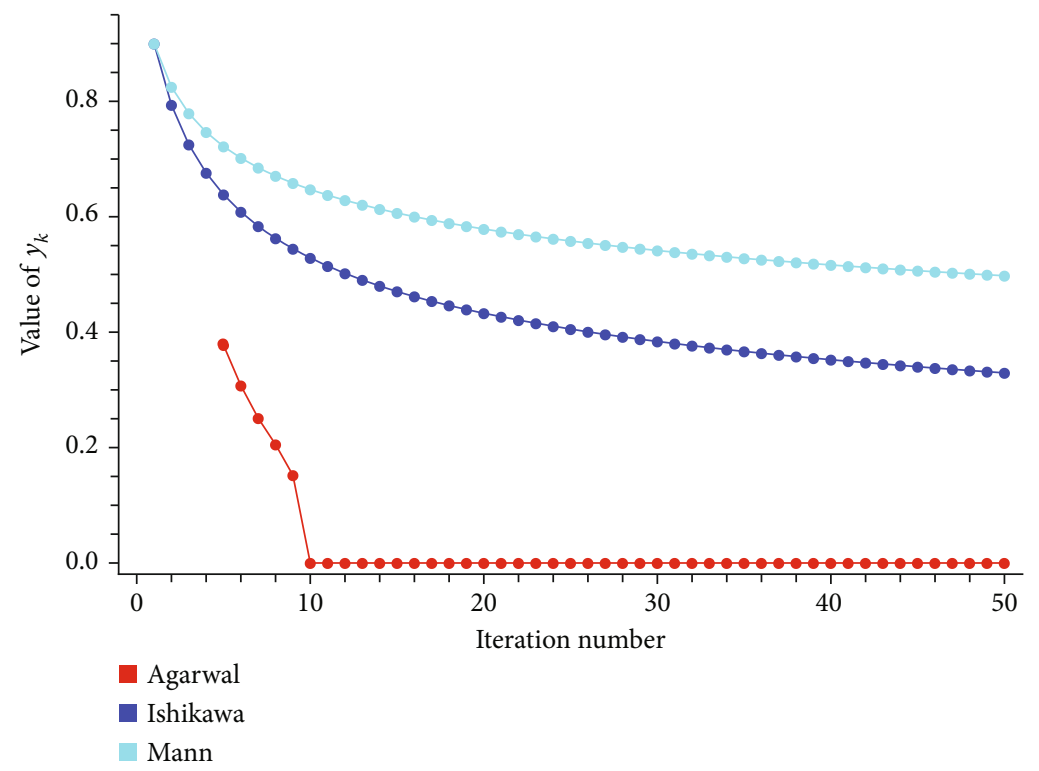

FIGURE 1: $\alpha_{k}=1 / k+1, \beta_{k}=k / k+1$.

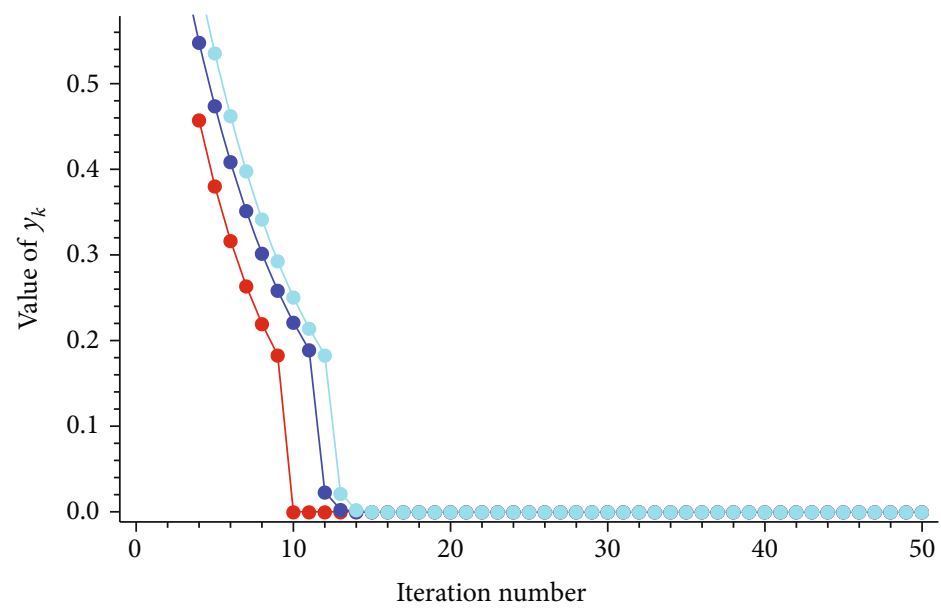

Agarwal

- Ishikawa

- Mann

Figure 2: $\alpha_{k}=1-1 / \sqrt{6 k+1}, \beta_{k}=1 / k^{3}$.

$=5 s^{\prime} / 6$. Thus,

$$
\begin{aligned}
q\left(s, \mathscr{L} s^{\prime}\right) & \leq q(s, \mathscr{L} s)+q\left(\mathscr{L} s, \mathscr{L}^{\prime}\right)=|s-\mathscr{L} s|+\left|\mathscr{L} s-\mathscr{L} s^{\prime}\right| \\
& =|s-\mathscr{L} s|+\left|\frac{5 s}{6}-\frac{5 s^{\prime}}{6}\right|=|s-\mathscr{L} s|+\frac{5}{6}\left|s-s^{\prime}\right| \\
& \leq|s-\mathscr{L} s|+\left|s-s^{\prime}\right| \leq 6|s-\mathscr{L} s|+\left|s-s^{\prime}\right| \\
& =6 q(s, \mathscr{L} s)+q\left(s, s^{\prime}\right) .
\end{aligned}
$$

So,

$$
\begin{aligned}
q\left(s, \mathscr{L} s^{\prime}\right) & =\left|s-\mathscr{L} s^{\prime}\right|=\left|s-\left(\frac{5 s^{\prime}}{6}\right)\right|=\left|\frac{6 s-5 s^{\prime}}{6}\right| \\
& =\left|\frac{s+5 s-5 s^{\prime}}{6}\right| \leq\left|\frac{s}{6}\right|+\left|\frac{5 s-5 s^{\prime}}{6}\right| \leq|s|+\left|s-s^{\prime}\right| \\
& =|s-\mathscr{L} s|+\left|s-s^{\prime}\right| \leq 6|s-\mathscr{L} s|+\left|s-s^{\prime}\right| \\
& =6 q(s, \mathscr{L} s)+q\left(s, s^{\prime}\right) .
\end{aligned}
$$

Case III. If $s \in S_{1}$ and $s^{\prime} \in S_{2}$. Then $\mathscr{L} s=0$ and $\mathscr{L} s^{\prime}=5 s^{\prime} / 6$. Case IV. If $s \in S_{2}$ and $s^{\prime} \in S_{1}$. Then $\mathscr{L} s=5 s / 6$ and $\mathscr{L} s^{\prime}=0$ and 


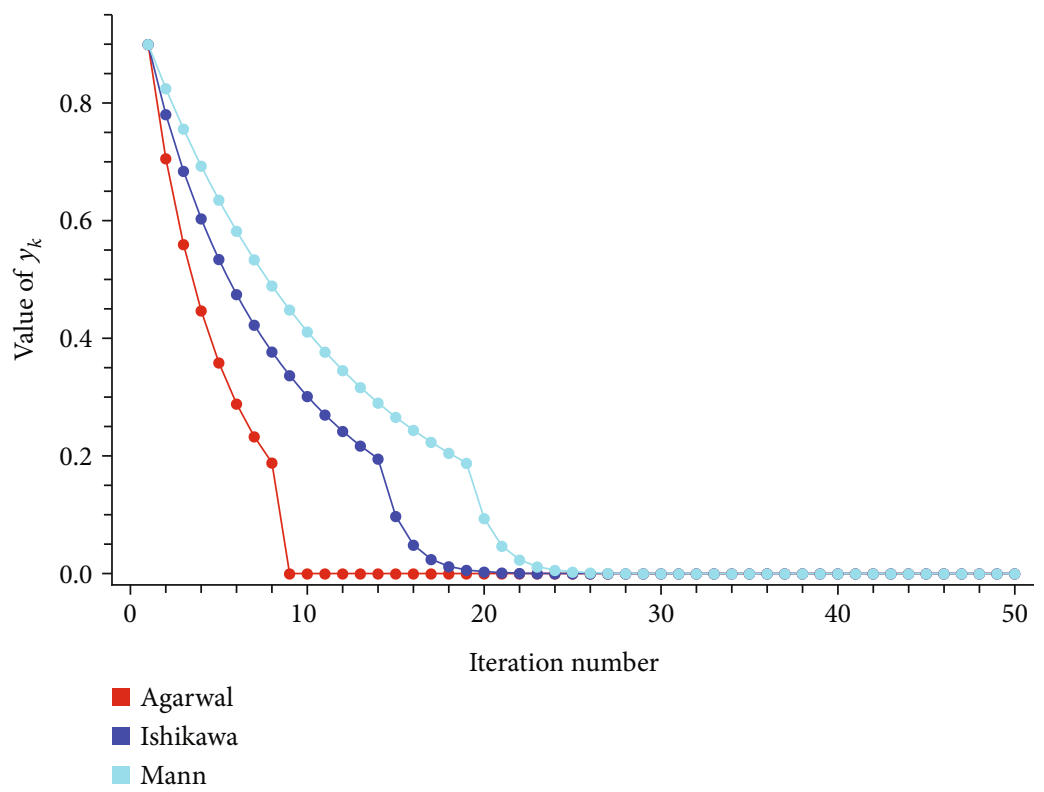

Figure 3: $\alpha_{k}=1 / 2, \beta_{k}=1 / \sqrt{k+1}$.

$$
\begin{aligned}
q\left(s, \mathscr{L} s^{\prime}\right)= & \left|s-\mathscr{L}^{\prime}\right|=|s|=6\left|\frac{s}{6}\right|=6|s-\mathscr{L} s| \leq 6|s-\mathscr{L} s| \\
& +\left|s-s^{\prime}\right|=6 q(s, \mathscr{L} s)+q\left(s, s^{\prime}\right) .
\end{aligned}
$$

Next, we provide the values for which $\mathscr{L}$ does not satisfy condition $(C)$. Choose $s=1 / 8$ and $s^{\prime}=1 / 5$. It is easy to show $1 / 2 d(s, \mathscr{L} s)<q\left(s, s^{\prime}\right)$, but $q\left(\mathscr{L} s, \mathscr{L} s^{\prime}\right)>q\left(s, s^{\prime}\right)$. For any choice of $k \in \mathbb{N}$, let $\alpha_{k}=0.70$ and $\beta_{k}=0.65$, then, the observations are given in Table 1 .

Now, we study the behavior of Agarwal, Ishikawa, and Mann iterations under different cases. Notice that $q\left(y_{k}, \omega\right)$ $<10^{-10}$ is the stopping criteria, $y_{1}=0.9$, and we stop the iterates for $k=50$. In this case, Figures 1,2 , and 3 show that the Agarwal iteration process converges faster to the fixed point $\omega=0$ as compared to the other iterations.

\section{Conclusions}

In this paper, we have initiated the finding of fixed points for the generalized class of mappings due to Garcia-Falset et al. [10] in the general setting of 2-UCHS. We established several strong and $\Delta$-convergence theorems under the Agarwal iterative process [8]. We have presented a new example of mapping having condition $(E)$ but not $(C)$ and proved that its Agarwal iterative process [8] is more effective than the corresponding Mann [13] and Ishikawa [12] iterative processes. In view of the above discussion, our results simultaneously hold in UCBS, $\mathrm{CAT}(0)$, and some $\mathrm{CAT}(\kappa)$ spaces. The present results can be extended and applied to many fields of applied sciences and differential equations. Thus, our results extend the corresponding results of Bagherboum [11], Phuengrat- tana [7], Basarir and Sahin [9], and Nanjaras et al. [6] from the general setting of domains and faster iteration process.

\section{Data Availability}

The data used to support the findings of this study are included in the references within the article.

\section{Conflicts of Interest}

The authors declare no conflict of interest.

\section{Authors' Contributions}

All authors contributed equally and significantly in writing this article. All authors read and approved the final manuscript.

\section{Acknowledgments}

The authors are grateful to the Spanish Government for Grant RTI2018-094336-B-I00 (MCIU/AEI/FEDER, UE) and to the Basque Government for Grant IT1207-19.

\section{References}

[1] F. E. Browder, "Nonexpansive nonlinear operators in a Banach space," Proceedings of the National Academy of Sciences of the United States of America, vol. 54, no. 4, pp. 1041-1044, 1965.

[2] W. A. Kirk, "A fixed point theorem for mappings which do not increase distances," The American Mathematical Monthly, vol. 72, no. 9, pp. 1004-1006, 1965.

[3] D. Gohde, "Zum Prinzip der Kontraktiven Abbildung," Mathematische Nachrichten, vol. 30, no. 3-4, pp. 251-258, 1965.

[4] W. A. Kirk, "Geodesic geometry and fixed point theory," in Seminar of Mathematical Analysis, Colecc, Abierta: Malaga, Spain, 2003. 
[5] T. Suzuki, "Fixed point theorems and convergence theorems for some generalized nonexpansive mappings," Journal of Mathematical Analysis and Applications, vol. 340, no. 2, pp. 1088-1095, 2008.

[6] B. Nanjaras, B. Panyanak, and W. Phuengrattana, "Fixed point theorems and convergence theorems for Suzuki-generalized nonexpansive mappings in CAT(0) spaces," Nonlinear Analysis: Hybrid Systems, vol. 4, no. 1, pp. 25-31, 2010.

[7] W. Phuengrattana, "Approximating fixed points of Suzukigeneralized nonexpansive mappings," Nonlinear Analysis: Hybrid Systems, vol. 5, no. 3, pp. 583-590, 2011.

[8] R. P. Agarwal, D. O'Regon, and D. R. Sahu, "Iterative construction of fixed points of nearly asymtotically non-expansive mappings," Journal of Nonlinear and convex Analysis, vol. 8, pp. 61-79, 2007.

[9] M. Basarir and A. Sahin, "On the strong and $\Delta$-convergence of $S$-iteration process for generalized nonexpansive mappings on CAT(0) spaces," Thai Journal of Mathematics, vol. 12, pp. 549-559, 2014.

[10] J. Garcia-Falset, E. Llorens-Fuster, and T. Suzuki, "Fixed point theory for a class of generalized nonexpansive mappings," Journal of Mathematical Analysis and Applications, vol. 375, no. 1, pp. 185-195, 2011.

[11] M. Bagherboum, "Approximating fixed points of mappings satisfying condition (E) in Busemann space," Numerical Algorithms, vol. 71, pp. 25-39, 2011.

[12] S. Ishikawa, "Fixed points by a new iteration method," Proceedings of American Mathematical Society, vol. 44, no. 1, pp. 147-150, 1974.

[13] W. R. Mann, "Mean value methods in iteration," Proceedings of the American Mathematical Society, vol. 4, no. 3, pp. 506$510,1953$.

[14] L. Leustean, "A quadratic rate of asymptotic regularity for CAT(0)-spaces," Journal of Mathematical Analysis and Applications, vol. 325, no. 1, pp. 386-399, 2007.

[15] T. Laokul and B. Panyanak, "A generalization of the $(\mathrm{CN})$ inequality and its applications," Carpathian Journal of Mathematics, vol. 36, no. 1, pp. 81-90, 2020.

[16] M. A. Khamsi and A. R. Khan, "Inequalities in metric spaces with applications," Nonlinear Analysis, vol. 74, no. 12, pp. 4036-4045, 2011.

[17] H. K. Xu, "Inequalities in Banach spaces with applications," Nonlinear Analysis, vol. 16, no. 12, pp. 1127-1138, 1991.

[18] L. Leustean, "Nonexpansive iterations in uniformly convex Whyperbolic spaces," in Nonlinear Analysis and Optimization I. Nonlinear Analysis, vol. 513 of Contemporary Mathematics, pp. 193-210, American Mathematical Society, Providence, RI, USA, 2010.

[19] W. A. Kirk and B. Panyanak, "A concept of convergence in geodesic spaces," Nonlinear Analysis: Theory, Methods \& Applications, vol. 68, no. 12, pp. 3689-3696, 2008.

[20] S. Dhompongsa, W. A. Kirk, and B. Panyanak, "Nonexpansive set-valued mappings in metric and Banach spaces," Journal of Nonlinear and Convex Analysis, vol. 8, pp. 35-45, 2007.

[21] H. F. Senter and W. G. Dotson, "Approximating fixed points of nonexpansive mappings," Proceedings of American Mathematical Society, vol. 44, no. 2, pp. 375-380, 1974. 